This item was submitted to Loughborough's Research Repository by the author.

Items in Figshare are protected by copyright, with all rights reserved, unless otherwise indicated.

\title{
Human wetness perception of fabrics under dynamic skin contact
}

PLEASE CITE THE PUBLISHED VERSION

https://doi.org/10.1177/0040517517716905

PUBLISHER

SAGE Publications (UK and US)

VERSION

AM (Accepted Manuscript)

PUBLISHER STATEMENT

This work is made available according to the conditions of the Creative Commons Attribution-NonCommercialNoDerivatives 4.0 International (CC BY-NC-ND 4.0) licence. Full details of this licence are available at: https://creativecommons.org/licenses/by-nc-nd/4.0/

\section{LICENCE}

CC BY-NC-ND 4.0

\section{REPOSITORY RECORD}

Raccuglia, Margherita, K. Pistak, Christian Heyde, J. Qu, N. Mao, Simon Hodder, and George Havenith. 2017. "Human Wetness Perception of Fabrics Under Dynamic Skin Contact". figshare.

https://hdl.handle.net/2134/25208. 


\title{
Human wetness perception of fabrics under dynamic skin contact
}

\begin{abstract}
This experiment studied textile (surface texture (ST), thickness) and non-textile (local skin temperature $\left(\mathrm{T}_{\mathrm{sk}}\right)$ changes, stickiness sensation and fabric-to-skin pressure) factors affecting skin wetness perception (WP) under dynamic interactions. Changes in fabric texture sensation between WET and DRY state and their effect on pleasantness were also studied. ST of eight fabric samples, selected for different structures, was determined from surface roughness measurements using the Kawabata Evaluation System (KES). Sixteen participants assessed fabric WP, at high pressure (HIGH-P) and low pressure (LOW-P) conditions, stickiness, texture and pleasantness sensation on the ventral forearm. Differences in WP $(p<0.05)$ were not determined by texture properties and/or texture sensation. Stickiness sensation and local $\mathrm{T}_{\text {sk }}$ drop were determined as predictors of WP $\left(\mathrm{r}^{2}=0.89\right)$, and although thickness did not correlate with WP directly when combined with stickiness sensation it provided a similar predictive power $\left(\mathrm{r}^{2}=\right.$ 0.86). Greater $(\mathrm{p}<0.05)$ WP responses in HI-P were observed compared with LOW-P. Texture sensation affected pleasantness in DRY $\left(r^{2}=0.89\right)$ and WET $\left(r^{2}=0.93\right)$. In WET, pleasantness was significantly reduced $(\mathrm{p}<0.05)$ compared to DRY, likely due to the concomitant increase in texture sensation ( $p<0.05$ ). In summary, under dynamic conditions, changes in stickiness sensation and WP could not be attributed to fabric texture properties (i.e. surface roughness) measured by the Kawabata Evaluation System. In dynamic conditions thickness or skin temperature drop can predict fabric WP only when including stickiness sensation data.
\end{abstract}

\section{Key words}

Wetness perception, fabric motion, stickiness, surface roughness, fabric texture, Kawabata, pleasantness 


\section{Introduction}

Whenever we increase our activity level and body heat content, sweat production causes moisture to build-up on the skin. The human ability to perceive skin wetness causes tactile and thermal discomfort (Fukazawa and Havenith 2009), this driving behavioural thermoregulatory responses (Schlader et al. 2010) aimed at maintaining homeostasis, ensuring health and survival (Parsons 2002). In absence of visual or auditory cues, skin wetness is perceived via learning processes (Bentley 1900) and through the central integration of thermal and mechanical stimuli occurring at the skin (Bergmann Tiest et al. 2012; Filingeri and Havenith 2015).

A large body of research has been focusing on the complex multisensory modality of wetness perception using fabrics (Sweeney and Branson 1990a, b; Jeon et al. 2011; Niedermann and Rossi 2012). For instance, Li (2005), in wear trials, and recently Raccuglia et al. (2016), in local body sensorial trials, highlighted the contribution of cold sensation to the perception of fabric moisture. Specifically, in both studies greater wetness perception was observed in response to greater reduction in skin temperature, which in return was affected by fabric water content. By studying the contribution of each single sensory modality (thermo- and mechano- sensation), Bergman Tiest et al. (2012) concluded that when interacting statically with a wet fabric the only cue available to perceive wetness is the thermal one. Conversely, it has been recently shown that some mechanical cues (fabric pressure on the skin) also affect perceived wetness in 
static contact (upper back) (Raccuglia et al. 2016). In fact, in heavier fabrics the higher resultant skin pressure causes higher wetness perception responses, compared to lighter fabrics, despite having the same water content (Raccuglia et al. 2016). On the other hand, under dynamic contact (fabric manipulation) the mechanical cue, i.e. stickiness, can improve a person's ability to discriminate various wetness intensities (Bergmann Tiest et al. 2012).

The neurophysiological basis of wetness perception has been well documented in the classical work conducted by Bentley (1900), and has seen a revival in the last decade (Bergmann Tiest et al. 2012; Filingeri and Havenith 2015). However, in order to improve moisture sensation and thermal comfort of clothing it would be of great value to identify the textile parameters that trigger cutaneous thermal and mechanical inputs underpinning wetness perception. In addition, as single textile properties have often been defined using a whole range of physical tests, it would be of practical value to know which of these test parameters has the best predictive power for wetness perception. Only recently, the role of fabric thickness as factor determining wetness perception in saturated or in part saturated fabrics, under static skin contact has been demonstrated (Raccuglia et al. 2016). Nevertheless, under dynamic contact other fabric parameters might also play a role. In dynamic conditions the presence of moisture increases fabric to skin friction (Gwosdow et al. 1986; Kenins 1994; Sivamani et al. 2003), sensed as higher stickiness and used as cue to perceive wetness. 
The mechanical and surface properties of fabrics have been studied in the context of end-users choice and satisfaction, leading to a series of investigations looking at the relation between objective and subjective assessments (Alimaa et al. 2000; Cardello et al. 2003; Sular and Okur 2007). On the other hand, in the current study fabric texture properties will be evaluated to assess whether these influence the tactile cues underlying skin wetness perception. In this scenario, we hypothesised that, due to a greater number of contact points with the skin, fabrics with smoother surface texture will cause higher skin friction and/or displacement and will be perceived as wetter than fabrics with rougher surface texture. Following from a study focusing on the static interaction between the skin and fabrics, in the current experiment we 1) sought to identify the role of textile factors, such as surface texture and thickness as well as non-textile factors, i.e. local skin temperature changes and stickiness sensation on wetness perception under dynamic skin contact. Additionally, (2) we aimed to observe changes in fabric pleasantness and texture sensation between dry and wet state.

\section{Methods}

\section{Participants}

Sixteen young (yrs. $22.4 \pm 2.5$ ) male (8) and female (8) participants, of Western European and North American origins, with no history of sensory related disorders and active at least 4-6 hours per week, volunteered to participate in this study. The test 
procedure and instruments were explained to each participant verbally and through a written information form. Following from this, participants gave written informed consent for participation. Due to the nature of the study, participants were not informed on the detailed aim of the study, experimental conditions, magnitude of the stimuli (amount of water applied) or type of fabric. The protocol and procedures involved were approved by Loughborough University Ethics Committee. The study was conducted within the confines of the World Medical Association Declaration of Helsinki for medical research using human participants.

\section{Specimen}

Eight knitted fabrics (120 x $100 \mathrm{~mm}$ ) selected for different structure, fibre type, surface texture properties, thickness, and treatments were included in this experiment (Table 1).

The fabrics were grouped in 3 main clusters according to their thickness characteristics (Table 1): low (0.56-0.60 mm; L), medium (0.90-1.00 mm; M) and high (2.10 mm; H). The results of this study will primarily be applied for the design of base-layers sportswear, usually presenting low thickness characteristics, therefore four fabric samples were included in L and only two fabric samples were included in both $\mathrm{M}$ and $\mathrm{H}$.

Within each thickness group the fabrics presented different surface texture (ST), measured as surface roughness (SMD) by the Kawabata Evaluation System (Kawabata 1980) (KES; higher ST corresponds to higher roughness) (Table 1). During the 
subjective assessments the face side of the experimental samples was tested only in wale direction; therefore the KES measurements were also performed in this direction and used for the estimation of ST.

The fabrics were coded according to thickness group ( $\mathrm{L}=$ low thickness; $\mathrm{M}=$ medium thickness, $\mathrm{H}=$ high thickness), fibre type ( $\mathrm{CO}=$ cotton; $\mathrm{PM}=$ polyester multichanneled fibre cross-section; $\mathrm{P}=$ polyester) and ST (approximated surface texture, determined by rounding up to a whole number). For instance, MP2 stands for Medium thickness group, Polyester and ST of $\sim 2$. Table 1 reports specifications of the experimental fabric samples. 
Table 1 Specifications of the experimental fabrics grouped according to low (L), medium (M) and high (H) thickness and presenting different surface texture (ST) measured as surface roughness (SMD) by the Kawabata Evaluation System. Water content was defined as equal water per volume of textile. The criterion for water content manipulation is reported in the 'Conditions' section.

\begin{tabular}{|c|c|c|c|c|c|c|c|c|}
\hline Fabrics & $\begin{array}{l}\text { Thickness } \\
\text { Group }\end{array}$ & $\begin{array}{c}\text { Thickness } \\
(\mathrm{mm})\end{array}$ & Fibre type & Structure & $\underset{(\mu \mathrm{m})}{\mathrm{ST}}$ & $\begin{array}{c}\text { Mass } \\
\left(\mathrm{g} \cdot \mathrm{m}^{-2}\right)\end{array}$ & $\begin{array}{c}\text { Material } \\
\text { description }\end{array}$ & $\begin{array}{c}\text { Water } \\
\text { content } \\
(\mu \mathrm{L})\end{array}$ \\
\hline \multicolumn{9}{|c|}{ LOW } \\
\hline $\mathrm{LCO} 4$ & & 0.60 & cotton & single jersey & $3.7 \pm 0.18$ & 140 & 'fuzzy' texture & 2400 \\
\hline LPM6 & & 0.56 & polyester & single jersey & $5.9 \pm 1.8$ & 160 & $\begin{array}{l}\text { multi-chanelled } \\
\text { fibre cross-section }\end{array}$ & 2400 \\
\hline LP3 & & 0.56 & polyester & single jersey & $2.6 \pm 0.4$ & 160 & plain surface & 2400 \\
\hline \multirow[t]{2}{*}{ LP6 } & & 0.60 & polyester & single jersey & $6.4 \pm 0.6$ & 160 & profiled surface & 2400 \\
\hline & MEDIUM & & & & & & & \\
\hline MP2 & & 0.90 & polyester & double jersey & $1.9 \pm 0.3$ & 130 & $\begin{array}{l}\text { silicon treated } \\
\text { 'silk-like surface' }\end{array}$ & 3600 \\
\hline \multirow[t]{2}{*}{ MP3 } & & 1.00 & polyester & double jersey & $2.7 \pm 0.2$ & 280 & $\begin{array}{l}\text { untreated 'standard } \\
\text { surface' }\end{array}$ & 4000 \\
\hline & HIGH & & & & & & & \\
\hline HP4 & & 2.10 & polyester & double jersey & $3.6 \pm 1.4$ & 330 & 'smooth' texture & 8400 \\
\hline HP15 & & 2.10 & polyester & $\begin{array}{l}\text { double jersey } \\
\text { french terry }\end{array}$ & $15.3 \pm 1.7$ & 330 & 'rough' texture & 8400 \\
\hline
\end{tabular}

\section{Experimental set up}

The experimental set up consisted of: a fabric sample, an adjustable chair where each participant was positioned and a fabric motion rig (Fig 1).

\section{Fabric sample}

To prevent water spreading across a fabric area larger than 120 x 100 mm, each long side of the experimental fabrics $(120 \times 100 \mathrm{~mm})$ was fitted to a non-wicking material 
(200 x $120 \mathrm{~mm}$ ). The two non-wicking materials together with the fabric, in between, formed the fabric sample.

\section{Fabric motion rig}

Each fabric sample (Fig 1) was placed in a custom-made linear motion rig. The sample was connected to a motor drive on one side and to a counterweight on the other side. The fabric sample could run over two rollers, creating a horizontal area of stimulation. Under this area, the right forearm of each participant was placed onto a height adjustable arm rest, such that the fabric touched the ventral forearm. The latter's setting was adjustable vertically to ensure equal pressure/ contact area in different size arms.

The ventral forearm was selected as body region of interest for practical reasons. In fact this body site allowed easy applications of the fabrics in relation to the design of the

motion rig, yet maintaining the comfort status of the participants during the trial. Additionally, it has been indicated that the ventral forearm presents the same sensitivity to cold as the upper back (Parsons 2002), therefore the results can be compared with the existing literature (Sweeney and Branson 1990a; Raccuglia et al. 2016).

A dividing wall was mounted onto the fabric motion rig, approximately half way between the forearm and the arm of each participant. With this setting the participants could not see the experimental textile samples before, during and after the application process, therefore any visual influence on the perceptual responses was prevented. 
Each fabric sample was pulled bi-directionally across the skin at a velocity of $0.02 \mathrm{~m} . \mathrm{s}^{-1}$. Two fixed levels of pressure were applied: $127 \mathrm{~Pa}$ (LOW-P) and $236 \mathrm{~Pa}$ (HI-P). The order of this two pressure conditions was counterbalanced (the method to measure fabric-to-skin pressure is reported in the Conditions section). The range of travel of each fabric was of $5 \mathrm{~cm}$ per stroke, with a total of 8 strokes per fabric, 4 toward the medial forearm and 4 toward the lateral forearm.

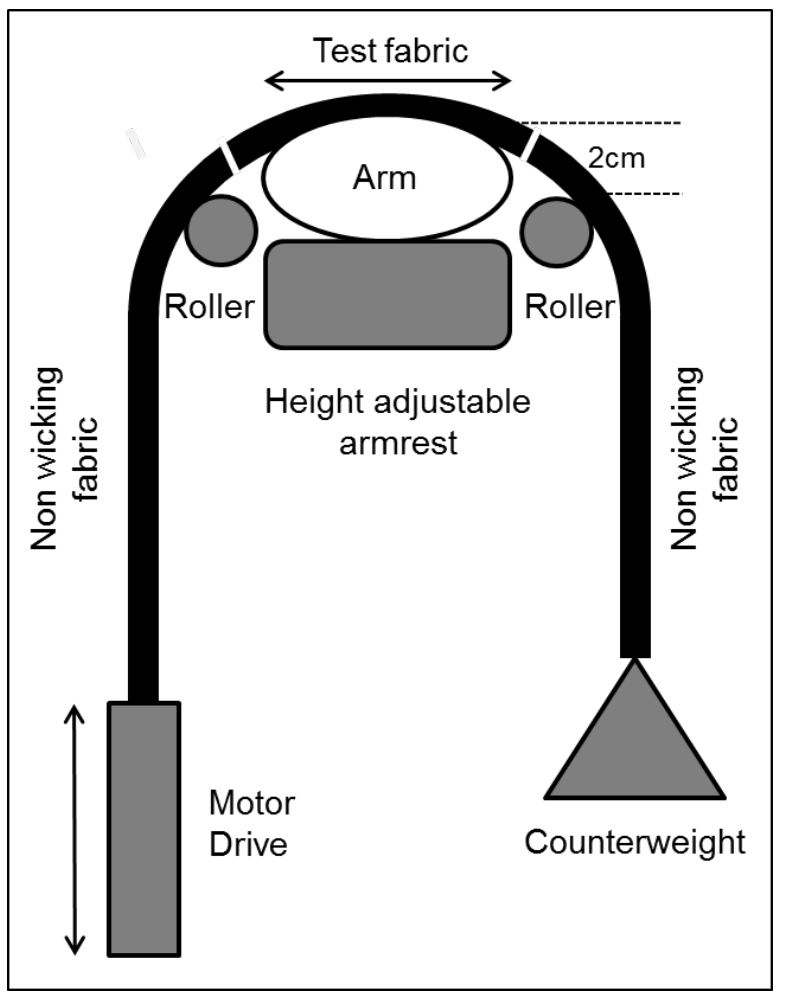

Figure 1. Schematic representation of the Fabric Motion Rig and experimental set up.

\section{Adjusting chair and participant position}


A chair was positioned at a standard distance from the fabric motion rig. After each participant settled on the chair, the investigator adjusted the position of the participant's right forearm on the armrest. The posterior margin of the olecranon was placed in line with the posterior edge of the armrest, the dorsal side of the forearm was located in contact with the armrest and the ventral side was left exposed, to allow the application of the samples. Because of individual forearm shape and size differences, to ensure standard level of contact between the sample and the ventral forearm, each forearm was maintained at a distance of $2 \mathrm{~cm}$ above the rollers of the motion rig. Additionally, the height of the chair was adjusted to achieve standard position of the forearm in respect to the arm ( $90^{\circ}$ angle), which varied based on the individual height of the participants.

\section{Conditions}

The fabric samples were tested in wet (WET) and dry (DRY) state. In WET, the samples were all treated with an amount of water corresponding to $50 \%$ of their total absorption capacity, according to the wetting procedure described in Raccuglia et al. (2016). This amount was shown to deliver the same quantity of water per unit of volume of the different fabrics.

Water absorption capacity was determined according to the 'water absorption capacity test' described by (Tang et al. 2014). For the test a fabric sample (100 x $100 \mathrm{~mm})$ was put into a tank of water and 5 min was allowed for it to sink completely into water. 
Following from this, the fabric was taken out by tweezers and hung onto a rod vertically until there was no water dripping within a 30 seconds interval. The water gain was calculated according to:

Water absorbed $(\mu L)=[\operatorname{wet} F(g)-\operatorname{dry} F(g)] * 1000 \frac{\mu L}{g}$

Where,

wetF, is the weight of the saturated fabric (g);

dryF, is the weight of the dry fabric (g).

The fabrics were wetted 30 min before the experimental trial, in accordance to the order (balanced) of application during the human sensorial assessment. Each fabric was positioned onto a plastic film and water was added by using a micropipette (SciQuip LTD, Newtown, UK) positioned at a fixed distance of $10 \mathrm{~cm}$ perpendicular to each sample and pointing at its centre. When the water was in equilibrium with the fabric, (specifically, when the water spread out uniformly across the sample; this took approximately 1 minute) each fabric was placed into a plastic bag which was securely sealed to prevent water evaporation. No water dripped from the samples inside the plastic bags during the storage period. Given that within each group the experimental samples had same thickness and same volume, the fabrics also presented the same 
relative to volume water content $\left(\mu \mathrm{L} \cdot \mathrm{mm}^{-3}\right)$ and same, or almost the same, absolute water content $\left(\mu \mathrm{L} \cdot \mathrm{mm}^{-2}\right)$ (Raccuglia et al. 2016) (Table 1).

The fabrics were also tested in the DRY condition to observe changes in texture and pleasantness sensation between WET and DRY. In the DRY condition no water was added to the fabric samples, which were in equilibrium regain with the environment (25 ${ }^{\circ} \mathrm{C}$ ambient temperature and $40 \%$ relative humidity). In DRY the pressure applied was of $127 \mathrm{~Pa}$ (same as LOW-P condition, see below).

To confirm the role of resultant fabric to skin pressure on wetness perception, as observed under static contact (Raccuglia et al. 2016), within the WET condition each fabric sample was tested at two pressure levels: low pressure of $127 \mathrm{~Pa}$ (LOW-P) and high pressure of $236 \mathrm{~Pa}$ (HIGH-P). The two pressure conditions were achieved using two different counterweights (200 g and $300 \mathrm{~g}$ ), attached at one end of each experimental sample, and mounted on the fabric motion operator rig (Fig 1). Extensive pilot testing were conducted to define the two resultant skin pressures. Results indicated that a pressure of $127 \mathrm{~Pa}$ represented the lowest possible pressure applicable in order to ensure enough tension in each WET fabric sample during the pulling process across the skin and to avoid sticking. The HIGH-P of $236 \mathrm{~Pa}$ was chosen with the aim of achieving perceivable differences from the LOW-P condition without applying excessive mechanical stimulation. Given the significantly higher weight of the two counterweights compared to the individual wet weight of each fabric sample (4.05-12.00 g), the effect 
of fabric weight on resultant skin pressure was negligible. To measure the pressure resulting from the application of each fabric sample plus attached clamp and counterweight, a calibrated electronic weighing scale (PSK 360-3, Kern, UK), with a maximum load of $360 \mathrm{~g}$ and a precision of $0.001 \mathrm{~g}$, was used. A cylinder with a forearm-like shape, made of hard foam, was placed on the measuring scale and each fabric, at both LOW-P and HIGH-P, was positioned on top of it. The two weight readings (g) were recorded and from these the two corresponding applied pressures (Pa), assuming a surface contact area of 10 x $10 \mathrm{~cm}$, were calculated according to:

Pressure applied $(\mathrm{Pa})=$ Weight reading $(\mathrm{kg}) * 9.81 \mathrm{~m} \cdot \mathrm{s}^{-2} /$ contact area $\left(\mathrm{m}^{2}\right)$.

\section{Study overview}

Fabrics were assessed in one single experimental trial including 3 different conditions: DRY; WET LOW-P; WET HIGH-P. Replicates of the 8 experimental fabric samples were tested under the 3 different experimental conditions, therefore a total of 24 fabric samples were tested during the experimental trial. New fresh fabric samples were used for each participant. The fabrics were assessed using a quantitative sensory test, which consisted of placing, in counter balanced order, the 24 samples on the right ventral forearm of each participant. Participants reported their local texture sensation, wetness perception, stickiness sensation and pleasantness sensation on ordinal scales (see Measurements section). Prior to the experimental trial, participants were familiarised 
( 15 min) with the experimental protocol, procedures and instruments used in the present study. The experimental trial was conducted immediately after the familiarisation session. The experiment was performed in a climate controlled room, maintained at air temperature at $25.8 \pm 0.2{ }^{\circ} \mathrm{C}$, relative humidity $39 \pm 0.7 \%$ and air velocity $<0.05 \mathrm{~m} / \mathrm{s}$ to ensure thermo-neutrality of the participants throughout the trial.

\section{Experimental protocol}

In the experimental trial participants entered the controlled climatic room and were positioned comfortably on the adjustable chair wearing standard T-shirt and shorts. Participants positioned their forearm on the armrest of the motion operator. A reference fabric sample (120 x $100 \mathrm{~mm}$ ) was placed on the skin, with the long sides of the sample perpendicular to the longitudinal axis of the forearm and two lines next to these two sides were drawn on the forearm to identify the fabrics' area of application. The centre of the sample was positioned 2/3 above the distal margin of the carpus, the length of the ventral forearm was $\sim 27 \mathrm{~cm}$ and it was measured from the distal margin of the carpus to the coronoid fossa. Participants were then instrumented with two thin skin temperature sensors (see Measurements section), in the skin area in contact with the fabric, and with temperature sensors across the body to measure body skin temperature. After this, participants rested for 20 min to allow time for skin temperature to stabilise. After the stabilisation period the investigator applied on the participants ventral forearm six reference fabrics, two for each ordinal scale, each corresponding to one of the two 
extreme points of texture, wetness and stickiness scale. The reference samples were chosen after extensive pilot studies. Specifically, a dry wool (very rough) and a dry silk (very smooth) materials were selected as references for texture sensation. Two samples of the same polyester fabric were used as 'extremely dry' (no water added) and as 'extremely wet' (50 \% of the total saturation) references. For stickiness, a wet silk fabric (extremely sticky) and a cotton fabric (not-sticky), both presenting same thickness and water content, were chosen as references. The score of each reference fabric was reported by the investigator who also informed the participants that the intensity of the subsequent fabrics would not exceed the range of the two references for each scale. Following from this, each experimental fabric was applied on the participants' ventral forearm, moving for a period of 20 seconds. Participants were alerted by the investigator before the application of each fabric. At the end of the 20 seconds, participants were encouraged to verbally report their texture sensation, wetness perception, stickiness sensation and pleasantness for the stimulated body area, using the four ordinal scales. After 15 seconds of application, local skin temperature was recorded. After 20 seconds the fabric sample was removed and a dry cloth was placed onto the tested body area to avoid any chilly sensation, consequent to the evaporation of any remaining water on the skin. The tested skin area was then gently wiped with the cloth and dried by blowing warm air; this took approximately 2 min and allowed temperature and hydration state of the skin to return to baseline before the application of the 
following experimental fabric. Additionally, since the repeated application of dynamic wet stimuli can decrease thermal and tactile sensitivity, 2 min of rest, before the subsequent fabric application, allowed the recovery of the sensory system. The same protocol was repeated for each of the 24 fabrics. Each experiment (stabilisation, familiarisation and experimental trial) took approximately 2 hours and participants were instructed to ask for a rest whenever they felt uncomfortable.

\section{Measurements}

\section{Surface texture}

To characterise the surface texture of the experimental fabrics surface roughness (SMD) was measured using the Kawabata Evaluation System (KES). For the measurement a sensor contacts the surface of the fabric under a constant normal force. The sensor consists of a metallic rod connected, in its freed end, to a thin wire with a U shape. Surface roughness is calculated from electrical signal generated by the vertical displacement of the sensor contacting the fabric surface.

\section{Skin temperature}

Local skin temperature during the contact with each fabric was measured with two fine wire (0.025 mm diameter, time constant of $0.003 \mathrm{sec}$.) type $\mathrm{T}$ thermocouples (RS Components, Northants, UK). The thermocouple temperatures were monitored and 
recorded every second throughout the application of the stimulus via a Grant Squirrel SQ2010 data logger (Grant Instrument Ltd., Cambridge, UK). Local skin temperature was calculated from the mean of the two measured spots. Local skin temperature drop (Local $\mathrm{T}_{\mathrm{sk}}$ Drop), resulting from the application of each wet fabric sample on the skin, was calculated according to:

Local $\mathrm{T}_{\mathrm{sk}}$ Drop $=$ PRE Local $\mathrm{T}_{\mathrm{sk}}-$ POST Local $\mathrm{T}_{\mathrm{sk}}$

Where:

PRE Local $\mathrm{T}_{\mathrm{sk}}$ is the local skin temperature before the application of the wet fabric (baseline) in ${ }^{\circ} \mathrm{C}$.

POST Local is $\mathrm{T}_{\mathrm{sk}}$ is the resultant local skin temperature recorded at second 15 during the application period in ${ }^{\circ} \mathrm{C}$.

Before testing, the thermocouples were calibrated by placing the measuring junction of each thermocouple in a circulating water bath whose temperature was monitored with a certified mercury thermometer.

To ensure thermo-neutrality, skin temperature of 5 body sites (check, abdomen, upper arm, lower back and back lower thigh) was measured throughout the experimental trial, with iButtons wireless temperature loggers (Maxim, San Jose, USA). From these five 
body sites, mean skin temperature, sampled every minute, was estimated according to the work of Houdas and Ring (1982).

\section{Texture}

To assess perception of fabric texture, i.e. roughness and smoothness, an ordinal bipolar, balanced scale was developed (Fig 2: A). To prevent forced choice, the scale had a neutral (0) point in the middle, corresponding to 'Neither rough nor smooth'. From zero to 9 (progressive increase in texture), the scale presented different levels of roughness, whereas from 0 to -9 (progressive reduction in texture) different magnitude of smoothness were displayed. During the scoring process participants were instructed to first associate the texture of the sample with one of the two attributes, i.e. rough (positive side) or smooth (negative side) and then to report the magnitude of the specific attribute chosen.

\section{Wetness}

A 30 points unipolar ordinal scale (Fig 2: B) was adopted to assess fabric wetness perception. The scale ranged from 0 to 30 , presenting descriptors at point $0,5,10,15$, 20, 25 and 30 (Raccuglia et al. 2016).

\section{Stickiness}


Sensations of fabric stickiness were assessed using a unipolar ordinal scale, ranging from 0 (Not-sticky) to 12 (Extremely sticky) and intermediate descriptors at point 3, 6 and 9 (Fig 2: C).

\section{Pleasantness}

A bipolar, balanced ordinal scale was developed to assess pleasantness sensation of the tested fabric samples (Fig 2: D). Same as the texture sensation scale, this scale presents an unforced choice at the middle point 0 (Neither pleasant nor unpleasant). Point $-2,-4$ and -6 were linked to the descriptors indicating progressive reduction in pleasantness, whereas points 2, 4 and 6 were linked to descriptors indicating progressive increase in pleasantness. 


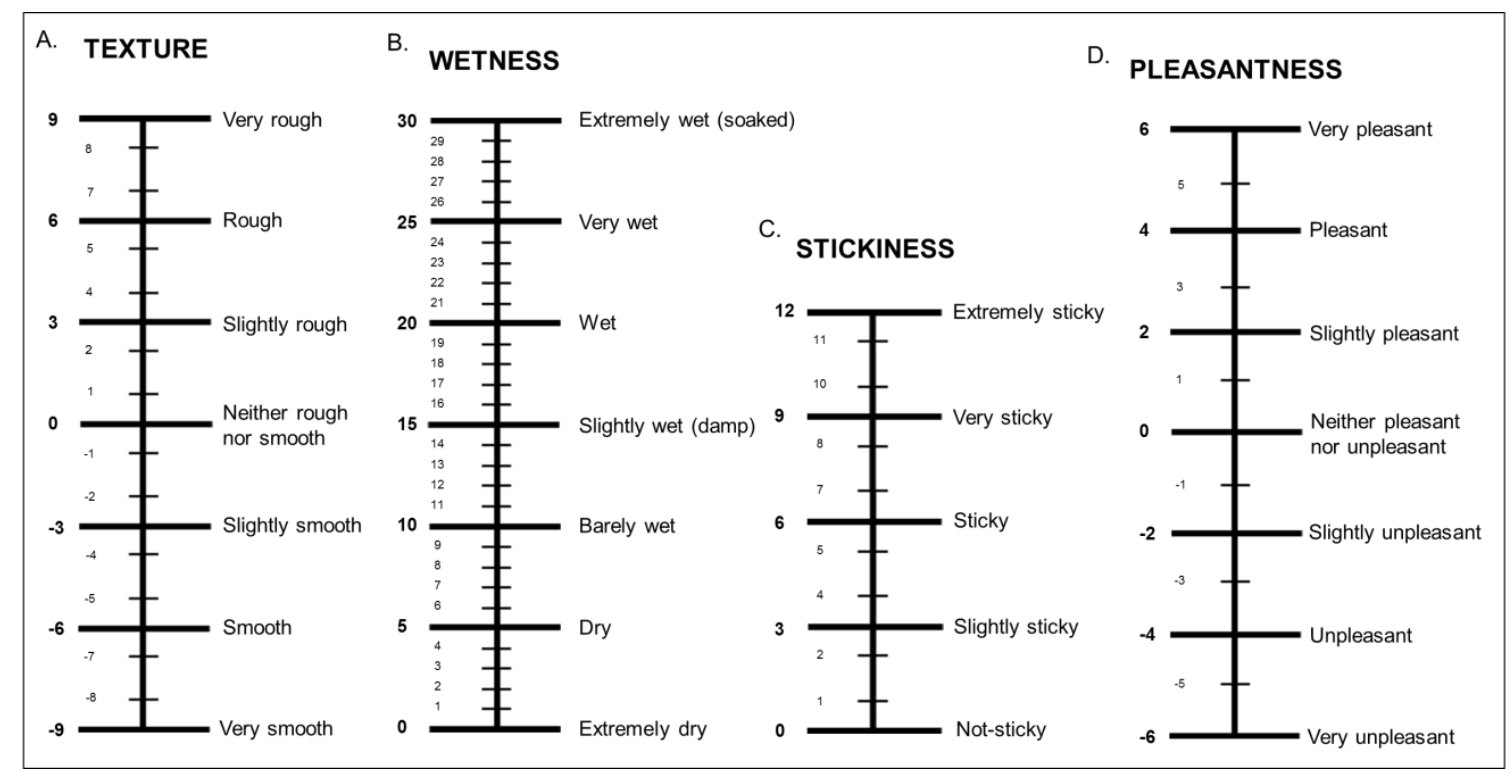

Figure 2: A texture sensation scale; 2: B wetness perception scale; 3: C stickiness sensation scale; 2: D pleasantness sensation scale.

\section{Statistics}

The independent variables were: fabric thickness, fabric surface texture, skin pressure (HIGH-P versus LOW-P), wet state (DRY versus WET). Dependent variables were: Local $T_{\text {sk }}$ Drop, mean $T_{\text {sk }}$, texture sensation, wetness perception, stickiness sensation and pleasantness.

Data were tested for normality of distribution and homogeneity of variance with Shapiro-Wilk and Levene’s tests, respectively. 
One way repeated measures ANOVA tests were conducted to assess whether mean $T_{\mathrm{sk}}$ was significantly different over time (T0-T55) and whether there were differences in local $\mathrm{T}_{\mathrm{sk}}$ Drop (normalised data from baseline) between fabric samples tested under WET.

Texture, wetness, stickiness and pleasantness sensation data were measured through means of ordinal scales and also violated the assumption of normality of distribution, therefore for the statistical analysis non-parametric tests were conducted.

The main effect of fabric surface texture on wetness perception (WET) was tested by Wilcoxon Signed Rank test for M and H (2 levels of comparisons; MP2 and MP3for M, HP4 and HP15 H) and by Friedman analysis of variance test for LOW (4 levels of comparisons; LCO4, LPM6, LP3, LP6. Friedman test was also conducted to test the main effect of fabric surface texture on texture sensation. Where significant effect was found post hoc analysis was conducted by Wilcoxon Signed Rank Test.

Wilcoxon Signed Rank tests were conducted to test the main effect of resultant fabric to skin pressure on wetness perception (2 levels of comparison for each fabric, i.e. LOW-P versus HIGH-P) and the main effect of wet state on texture sensation and pleasantness sensation (2 levels of comparison for each fabric, i.e. DRY versus WET)

Regression analyses were performed to observe the relations within and between objective (i.e. Local $\mathrm{T}_{\mathrm{sk}}$ Drop, fabric total water content, fabric thickness, surface 
texture) and subjective (i.e. wetness perception, stickiness sensation, texture sensation) variables, using data from group means. To choose the most suitable regression model, linear and second order polynomial analyses were performed for each subject. Individual $r^{2}$ values for linear and second order polynomial models were statistically compared using paired t-test. The regression model that explained the highest variance was then chosen for the analysis of group mean data.

In all analyses $\mathrm{p}<0.05$ was used to establish significant differences. Parametric data are reported as means \pm standard deviation (SD). Data were analysed using the software IBM SPSS Statistics (version 22) (IBM, USA).

\section{Results}

\section{Low pressure (LOW-P) condition}

Wetness perception

In L (0.56-0.60 mm thickness) sample LPM6 was perceived significantly dryer $(\mathrm{p}<$ 0.01) than the other three samples (LCO4, LP3, LP6), whereas none of these three samples (LCO4, LP3, LP6) significantly differed from each other (p > 0.05) (Fig 3, panel B).

In M (0.9-1.00 mm thickness) MP2 was perceived significantly wetter $(\mathrm{p}=0.008)$ than MP3 (Fig 3, panel B). 
No significantly different wetness perception responses were found in $\mathrm{H}(2.1 \mathrm{~mm}$ thickness) between HP4 and HP15 (

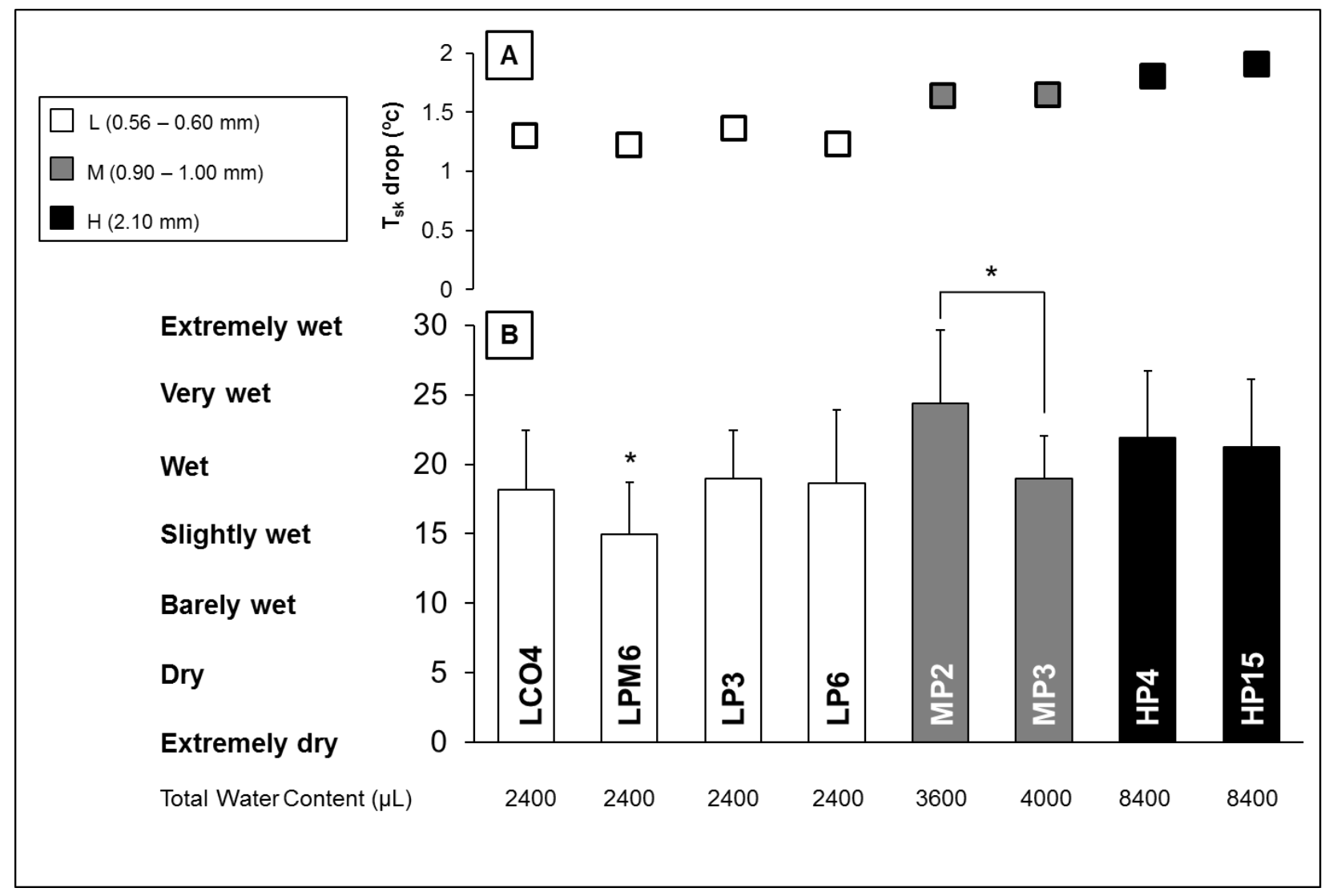

Figure 3 Panel A: average local $\mathrm{T}_{\text {sk }}$ (skin temperature) Drop from baseline (normalised data) in response to the dynamic application of each WET fabric sample in each thickness group: low (L), medium (M) and high $(H)$. Panel B: * Significant differences $(p<0.05)$ in wetness perception responses between fabrics within $\mathrm{L}$ or $\mathrm{M}$.

Mean and local skin temperature 
Mean skin temperature (sampled every minute), averaged over time, was $33.9 \pm 0.02{ }^{\circ} \mathrm{C}$ and did not significantly change $(\mathrm{p}<0.05)$ throughout the trial.

Baseline local $\mathrm{T}_{\mathrm{sk}}$ was $32.3 \pm 0.2{ }^{\circ} \mathrm{C}$ and was not significantly different $(\mathrm{p}<0.05)$ between each pre-application or condition (DRY, WET LOW-P, WET-HIGH-P).

Local $\mathrm{T}_{\mathrm{sk}}$ Drop (data normalised from baseline), in response to the application of the wet fabrics, was not significantly different within each thickness group: $p=0.85$ in $\mathrm{L}$ (0.56-0.60 mm thickness), $\mathrm{p}=0.89$ in $\mathrm{M}(0.9-1.0 \mathrm{~mm}$ thickness $)$ and $\mathrm{p}=0.90$ in $\mathrm{H}$ (Fig 3, panel A).

A positive relation was observed between wetness perception and Local $T_{\text {sk }}$ Drop $\quad\left(r^{2}=\right.$ 0.48, $\mathrm{p}=0.008)($ Fig 4). 


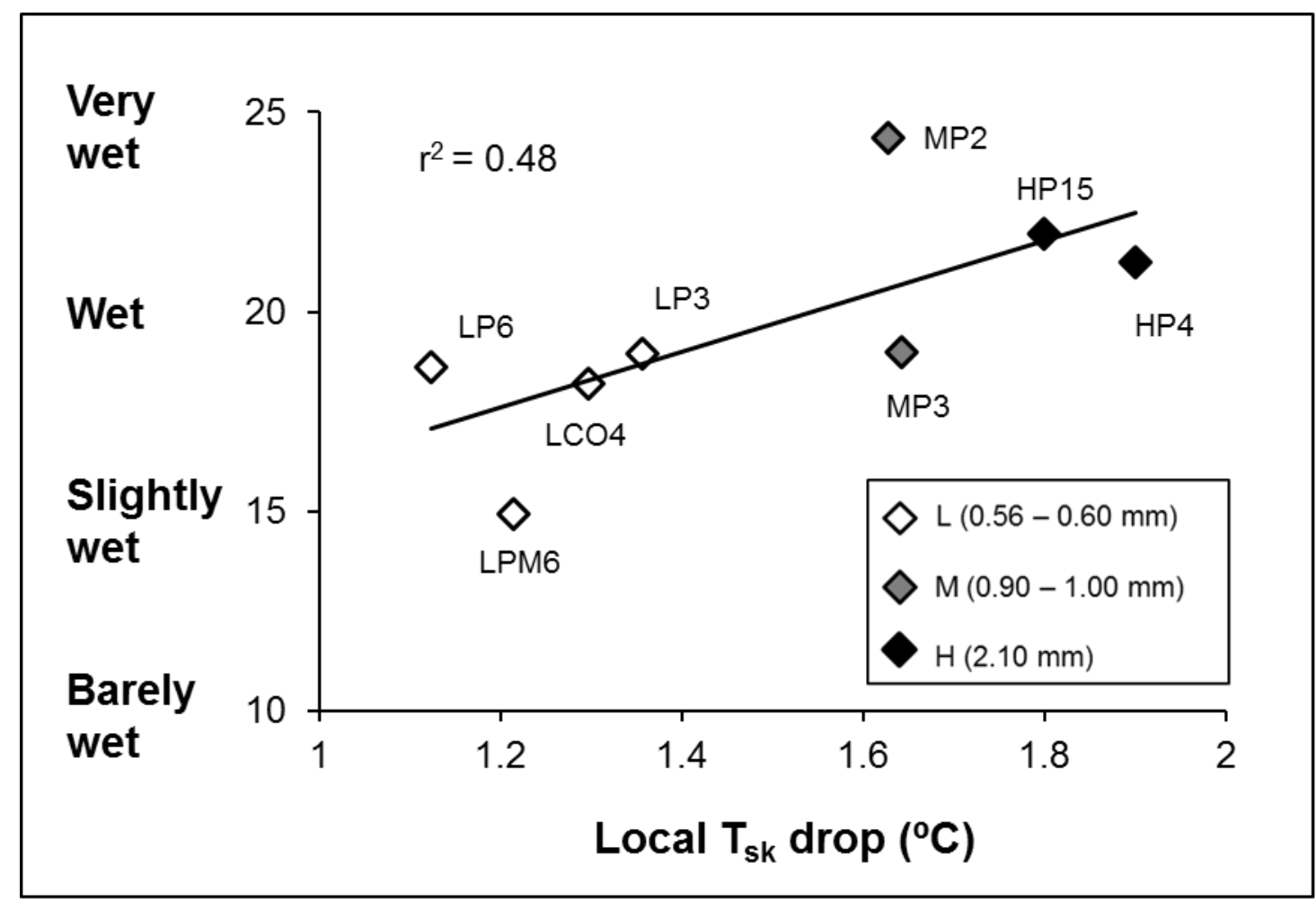

Figure 4: Relationship between wetness perception and local $\mathrm{T}_{\text {sk }}$ (skin temperature) drop in WET, LOW-P (low pressure) condition. Fabrics grouped according to L (low), M (medium) and high (H) thickness.

Relation between wetness perception and stickiness

A linear positive relation $\left(r^{2}=0.64, p=0.007\right)$ was observed between fabric wetness perception and stickiness sensation (Fig 5). Nevertheless, no relation was found between stickiness sensation and sample water content $\left(r^{2}=0.009, p=0.82\right)$. 


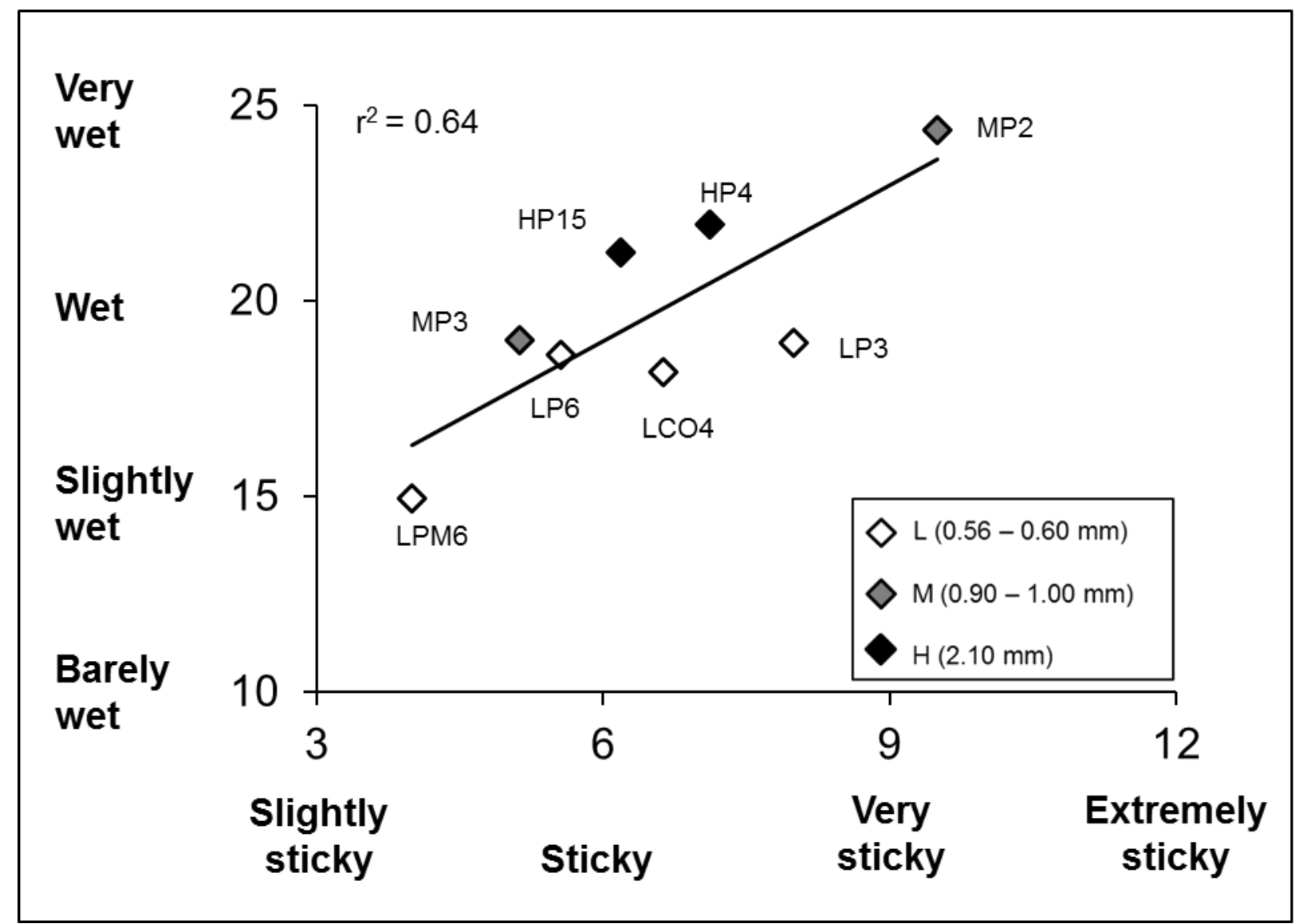

Figure 5: Relationship between wetness perception and stickiness sensation of the experimental fabrics tested in WET LOW-P (low pressure) condition. Fabrics grouped according to L (low), M (medium) and high $(\mathrm{H})$ thickness.

Relation of stickiness sensation and wetness perception with texture sensation and surface texture (ST)

In WET no relationship was observed between stickiness sensation and texture sensation $\left(r^{2}=0.087, p=0.48\right)$ nor between stickiness sensation and surface texture $\left(r^{2}=0.11, p=0.42\right)$ 
Wetness perception was not related to texture sensation $\left(r^{2}=0.003, p=0.96\right)$, neither to surface texture $\left(r^{2}=0.001, p=0.89\right)$.

Relation between wetness perception and fabric thickness

No significant relation was observed between wetness perception and fabric thickness $\left(r^{2}=0.29, p=0.166\right)($ Fig 6$)$. The lack of relation was mainly caused by fabric MP2 and LPM6, perceived as the driest and the wettest materials, respectively. In these two fabrics (LPM6 and MP2) the lowest and the highest wetness perception responses were not driven by their thickness or water content $\left(\mu \mathrm{L} \cdot \mathrm{mm}^{-3}\right)$, but rather to their resultant stickiness sensation (MP2 most sticky, LPM6 least sticky; Fig 5). 


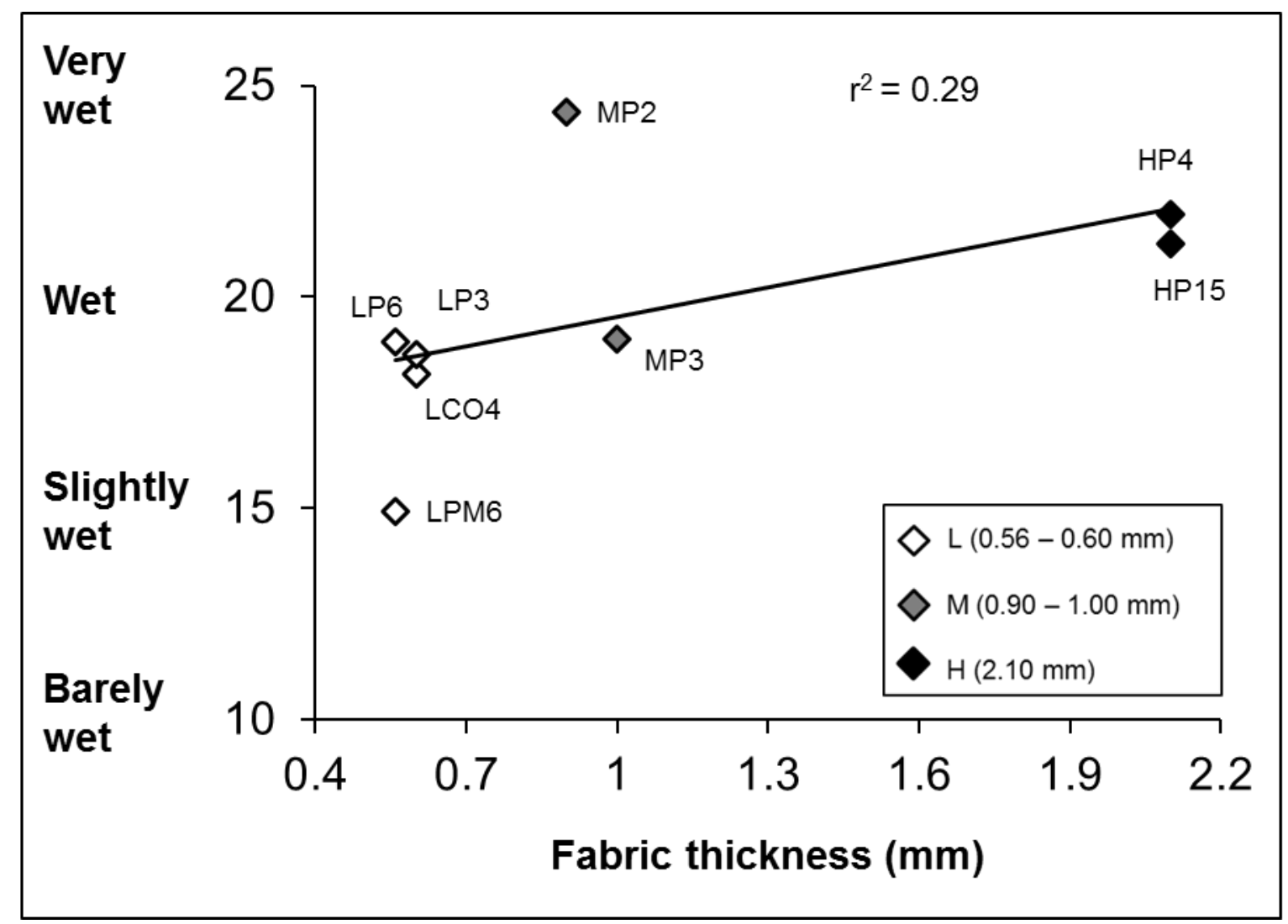

Figure 6: Relation between fabric wetness perception and fabric thickness in LOW-P (low pressure) condition. Fabrics grouped according to $L$ (low), M (medium) and high $(\mathrm{H})$ thickness.

Texture sensation and surface texture (ST)

In DRY a linear positive relation was observed between fabric texture sensation and surface texture $(\mathrm{ST})\left(\mathrm{r}^{2}=0.79, \mathrm{p}<0.005\right)$ (Fig 7a). The linear model was highly dominated by the roughest fabric HP15. When excluding this sample (HP15) from the model the relation appears less clear and only approaches significance $\left(r^{2}=0.55, p=\right.$ $0.06)$. 
In WET the relation between texture sensation and surface texture (ST) was less clear compared to DRY and only approached significance $\left(r^{2}=0.48, p=0.06\right)$ (Fig 7b). 


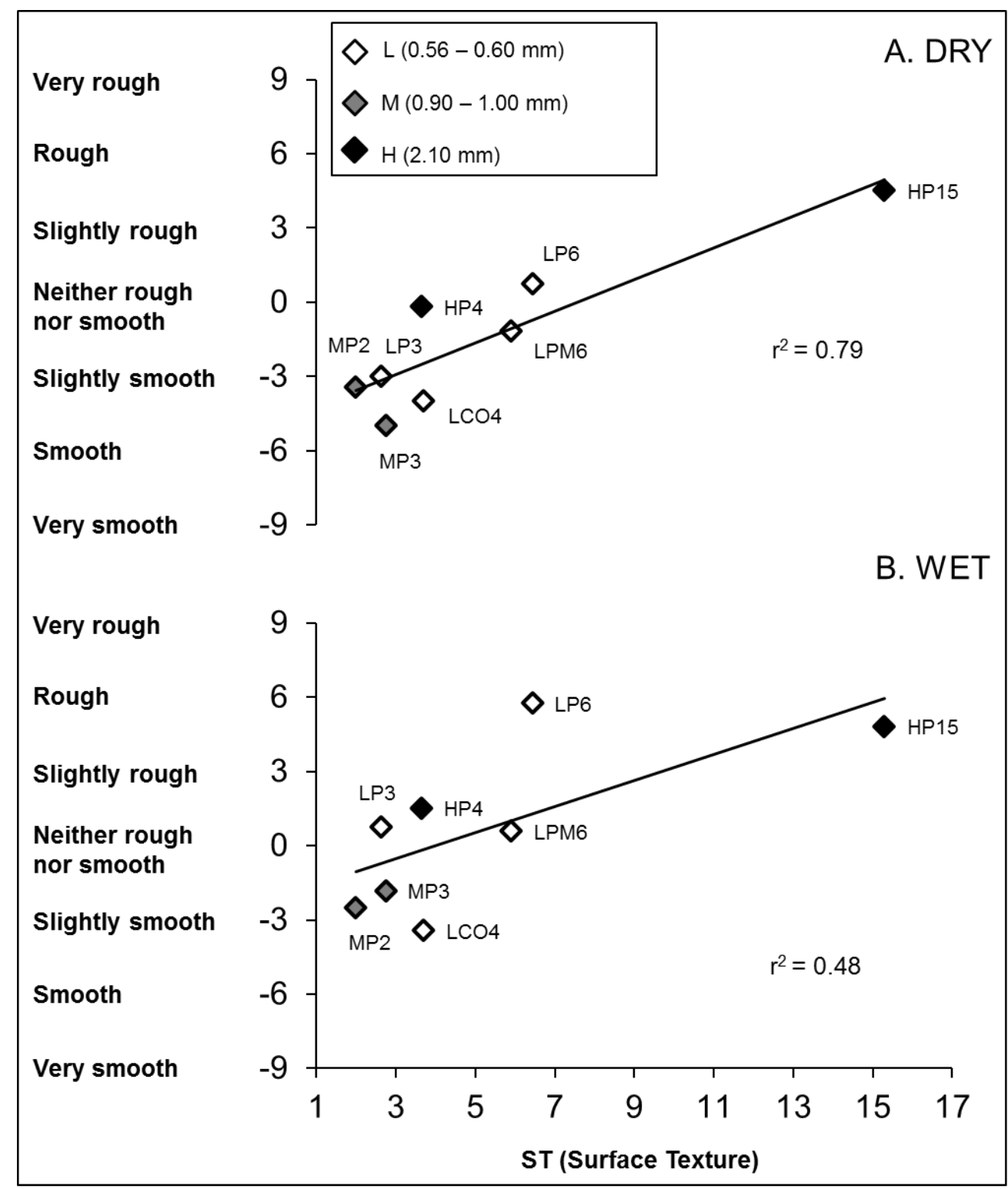

Figure 7a. Relationship between fabric texture sensation (in DRY) and surface roughness (ST), 7b relationship between fabric texture sensation assessed (in WET) and surface roughness (ST). Fabrics grouped according to $L$ (low), M (medium) and high $(\mathrm{H})$ thickness. 


\section{Wetness perception predictors}

In order to define factors affecting fabric wetness perception under dynamic contact, stepwise regression analysis was conducted. For this analysis textile factors, such as fabric thickness and surface texture as well as non-textile factors such as Local $\mathrm{T}_{\text {sk }}$ Drop and stickiness sensation, were imputed as independent variables and wetness perception as dependent variable.

Fabric surface texture and/or texture sensation did not appear as relevant predictors. Wetness perception was described by stickiness sensation and Local $\mathrm{T}_{\mathrm{sk}}$ Drop as the predicting variables (Table 2; MODEL 1), giving an explained variance of $89 \%$ :

$$
\text { Wetness perception }=4.338+\text { Stickiness } * 1.059+\text { Tsk drop } * 5.583
$$

Fabric stickiness sensation was the main predictor with a relatively larger Beta value at $0.64(\mathrm{p}=0.008)$, while local $\mathrm{T}_{\mathrm{sk}}$ drop was found to make a significant additional contribution to the predictive model $(\beta=0.53, \mathrm{p}=0.017)$.

Thickness alone did not predict wetness perception (Fig 6), mainly because of the latter's interaction with stickiness sensation. However, when replacing ' $\mathrm{T}_{\mathrm{sk}}$ drop' with 'thickness' and including 'stickiness sensation' a similar prediction model of the one above is obtained $\left(r^{2}=0.86, p=0.003\right)$ (Table 2, Model 2; Fig 8). 


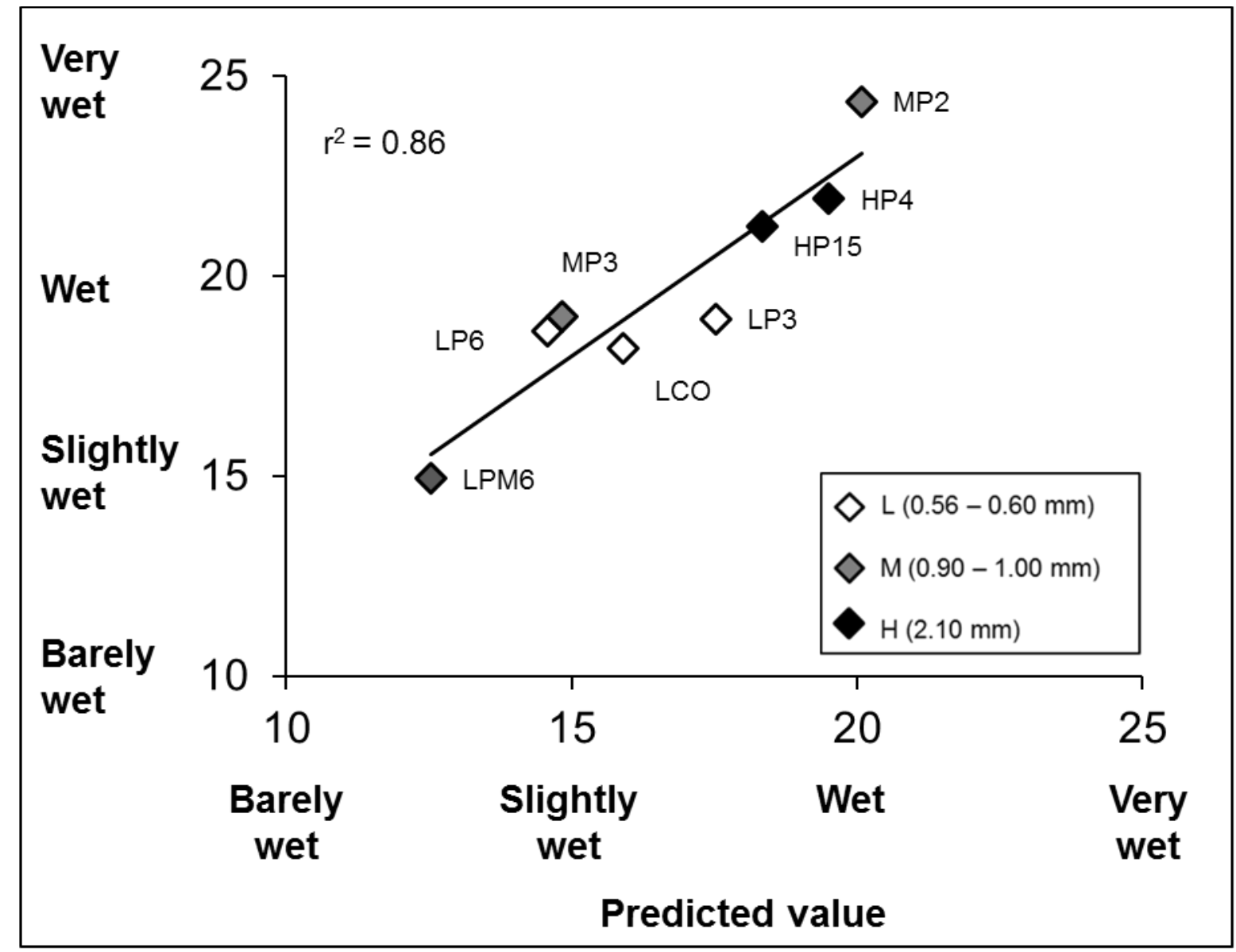

Figure 8 Representation of the prediction model of wetness perception (fabric stickiness sensation and fabric thickness as main predictors). Fabrics grouped according to L (low), M (medium) and high $(\mathrm{H})$ thickness. 
Table 2 Stepwise multiple regression analysis of wetness perception under LOW-P (low fabric to skin resultant pressure) condition.

\begin{tabular}{|c|c|c|c|c|c|c|c|c|}
\hline \multirow[b]{2}{*}{$\begin{array}{l}\text { Perceptual } \\
\text { variables }\end{array}$} & \multirow[b]{2}{*}{$\begin{array}{l}\text { Predictor } \\
\text { variables }\end{array}$} & \multicolumn{2}{|c|}{$\begin{array}{c}\text { Unstandardized } \\
\text { coefficient }\end{array}$} & \multicolumn{3}{|c|}{$\begin{array}{c}\text { Standardized } \\
\text { coefficient }\end{array}$} & \multirow[b]{2}{*}{$\mathrm{F}$} & \multirow[b]{2}{*}{$\mathrm{r}^{2}$} \\
\hline & & B & $\begin{array}{l}\text { SD } \\
\text { error }\end{array}$ & $\beta$ & $\mathrm{t}$ & Sign. & & \\
\hline \multirow{3}{*}{$\begin{array}{l}\text { MODEL } 1 \\
\text { Wetness } \\
\text { Perception }\end{array}$} & (Constant) & 4.338 & 2.693 & & 1.758 & .006 & \multirow[t]{3}{*}{22.051} & \multirow[t]{3}{*}{.89} \\
\hline & Stickiness & 1.059 & .247 & .642 & 4.290 & .008 & & \\
\hline & $\mathrm{T}_{\mathrm{sk}} \mathrm{drop}$ & 5.583 & .529 & .529 & 3.529 & .017 & & \\
\hline \multirow{3}{*}{$\begin{array}{l}\text { MODEL 2 } \\
\text { Wetness } \\
\text { Perception }\end{array}$} & (Constant) & 9.444 & 1.940 & & 4.869 & .005 & \multirow[t]{3}{*}{15.322} & \multirow[t]{3}{*}{.86} \\
\hline & Stickiness & 1.247 & .277 & .756 & 4.493 & .006 & & \\
\hline & Thickness & 1.985 & .717 & .466 & 2.770 & .039 & & \\
\hline
\end{tabular}

\section{High pressure (HIGH-P) condition}

Wetness perception scores

Perception data from the high pressure condition (HIGH-P) were typically higher but showed similar patterns to those obtained in the low pressure condition (LOW-P).

In L (low thickness group) LPM6 was again significantly dryer compared to the other three fabrics ( $p<0.001)$, LCO4, LP3 and LP6, whereas these three latter were not significantly different from each other ( $p>0.05$ ). In M (medium thickness group) MP2 was perceived significantly wetter than MP3 $(\mathrm{p}<0.05)$ whereas in H (high thickness group) HP4 and HP15 were not significantly different ( $\mathrm{p}>0.5)$. 
A linear relation was observed between WP and stickiness sensation $\left(r^{2}=0.79, p<0.05\right)$ whereas no correlation was observed between WP and thickness.

LOW-P and HIGH-P scores were compared to assess the role of resultant fabric to skin pressure on wetness perception. In HIGH-P samples were perceived significantly wetter ( $\mathrm{p}<0.05$ ) compared to the LOW-P condition, apart from LPM6 and MP2 in which the differences were not significant ( $p=0.318, p=0.975$, respectively) (Fig 9).

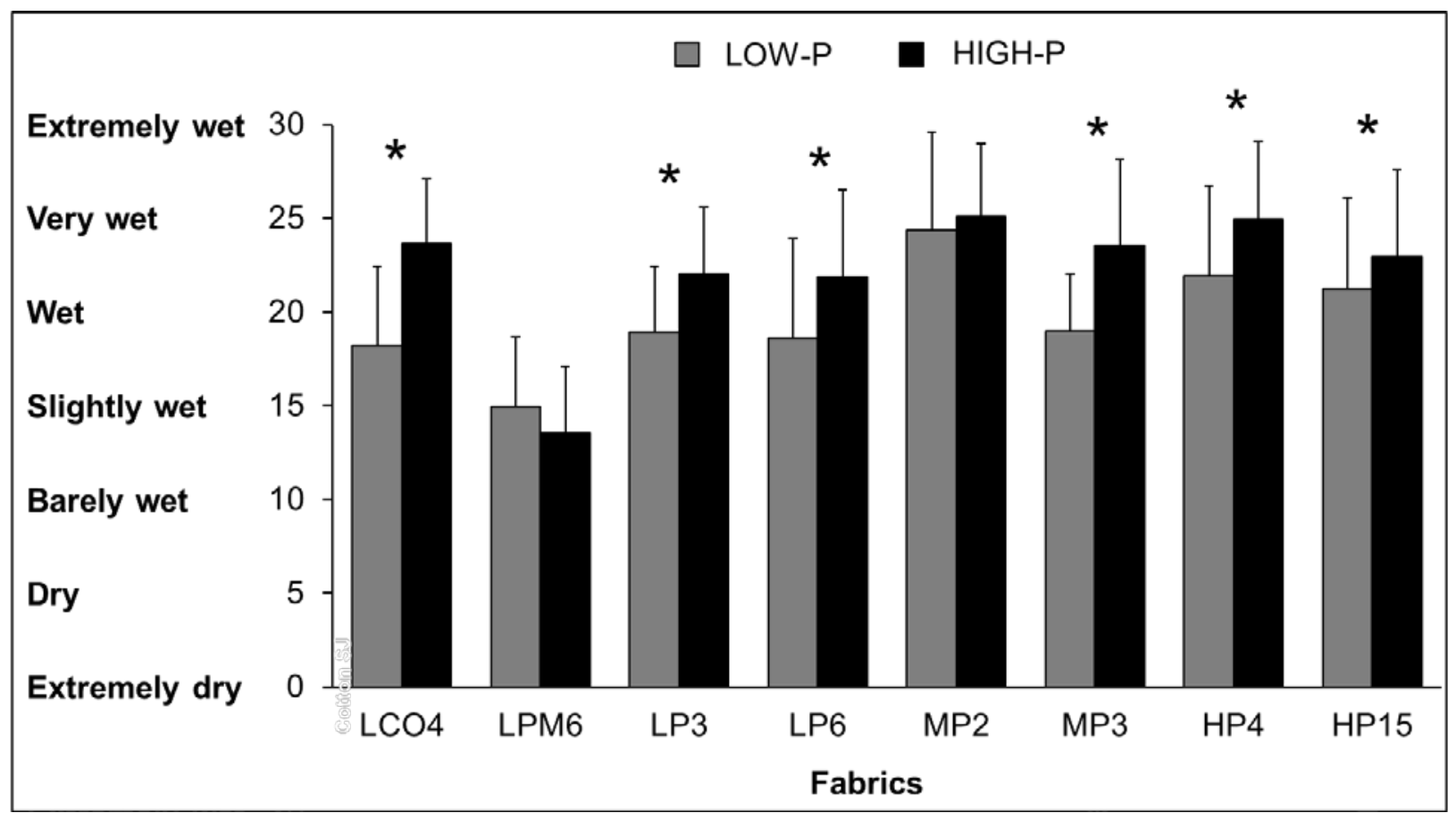

Figure 9 * Significant differences $(p<0.05)$ in fabric wetness perception between LOW-P (low pressure; grey bars) and HIGH-P (high pressure; black bars).

\section{Texture and pleasantness sensation}


Fabric pleasantness showed a significant relationship (second order polynomial fit) with texture sensation in both DRY $\left(r^{2}=0.93, p<0.001\right)$ and WET $\left(r^{2}=0.89, p<0.001\right)$, (Fig 10). Pleasantness was also significantly related (second order polynomial fit) with ST in DRY $\left(r^{2}=0.75, p<0.005\right)$ and WET $\left(r^{2}=0.39, p<0.05\right)$, although in WET the model presented a less predictive power.

Pleasantness sensation was significantly reduced in WET $(\mathrm{p}<0.05)$ compared to DRY, apart from LPM6 and HP15 which did not present significant differences between the two conditions ( $\mathrm{p}=0.53, \mathrm{p}=0.14$, respectively) .

Texture sensation increased in WET compared to DRY, however the increase was significant only in sample LP3 $(p=0.05)$, LP6 $(p=0.001)$ and MP3 $(p=0.03)$. 


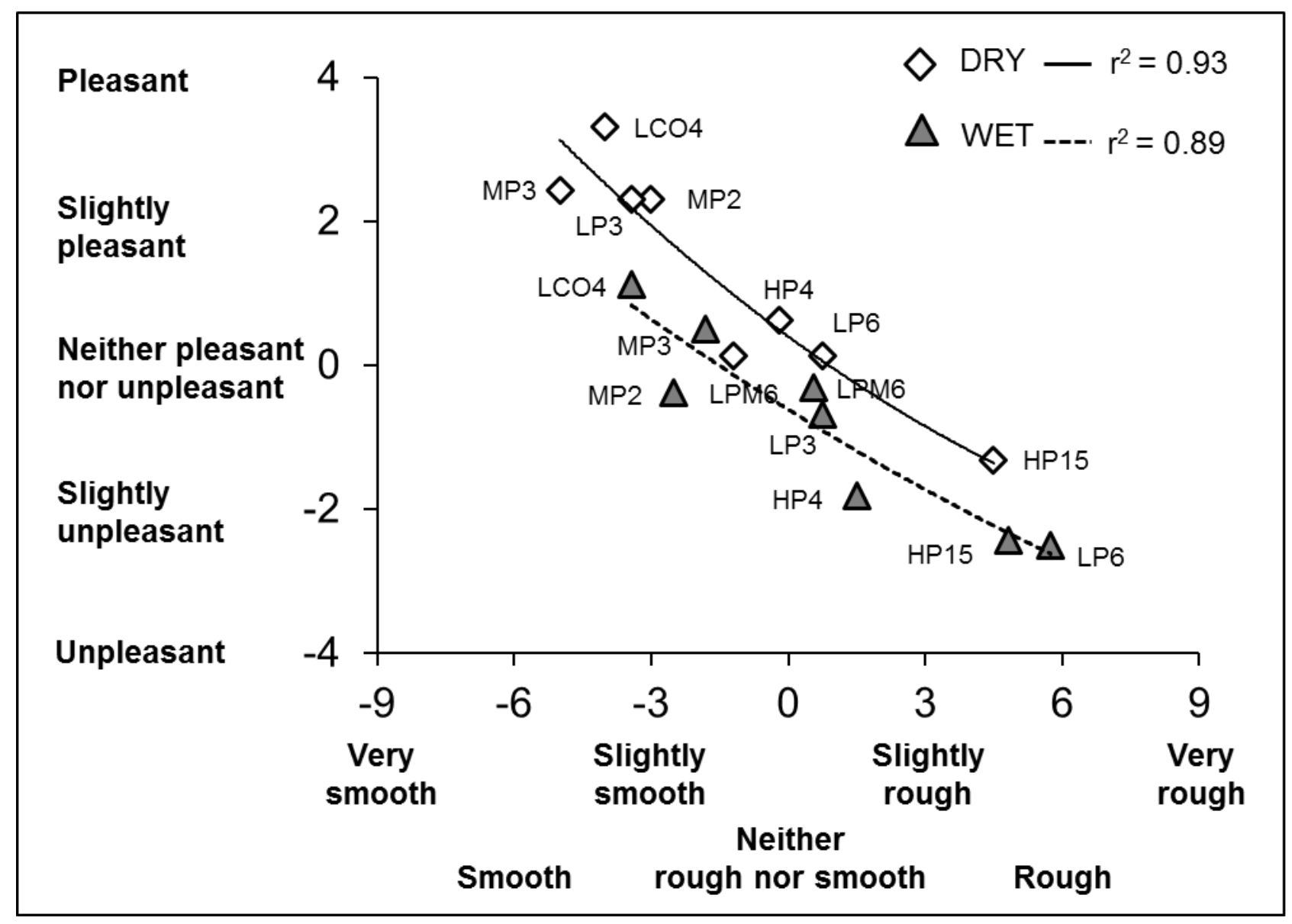

Figure 10 Relationship (second order polynomial fit) between fabric texture sensation and pleasantness sensation in DRY (diamonds symbols; solid curve) and WET (triangles symbols; dots curve) condition. Fabrics tested under LOW-P (low pressure) condition. 


\section{Discussion}

The aim of the current study was to identify the textile properties triggering cutaneous tactile and thermal inputs underpinning wetness perception in dynamic skin contact. In order to correct for volume/thickness differences in fabric water content, the same relative to volume water amount $\left(\mu \mathrm{L} \cdot \mathrm{mm}^{-3}\right)$ was applied, the latter corresponding to the $50 \%$ of the fabric's total saturation.

We hypothesised that, due to a greater number of contact points with the skin, fabrics with smoother surface texture will cause higher skin friction and/or displacement, sensed as higher stickiness and associated with greater wetness perception. Conversely to our research hypothesis, stickiness sensation and wetness perception did not show any correlation with the fabric texture property determined by the KES system and/or with texture sensation, and when conducting multiple regression analyses the latter were not identified as relevant predictors. Nevertheless, wetness perception was related with fabric stickiness sensation, therefore we could not totally reject our research hypothesis. In fact, we speculate that the lack of correlation was not due to a fundamental issue, but rather to a methodological issue, i.e. the Kawabata Evaluation system may not be an appropriate test method to predict stickiness sensation of wet fabrics.

With regard to wetness perception, the earlier observed relation to fabric thickness and skin cooling in static tests (triggering thermal responses; Raccuglia et al. 2016) was not 
observed here. However, when including stickiness sensation and therefore, correcting for the tactile responses, fabric thickness was shown to be a valid predictor and significantly contributed to the total variance (86 \%) in fabric wetness perception under dynamic skin contact too. Similarly, when selecting local skin temperature drop (thermal cue) together with stickiness sensation (tactile cue) as independent variables, an even better model of fabric wetness perception is obtained (explaining $89 \%$ of the total variance), indicating that the fabric thickness acts through its relation with the level of skin cooling based on the higher absolute water content of the thicker fabrics. In line with our previous work on static contact (Raccuglia et al. 2016), also in dynamic contact under conditions of higher fabric-to-skin pressure (triggering mechanical stimuli), greater wetness perception responses were observed. The latter suggests that fabric weight can have an effect on wetness perception.

Finally, comparisons of texture sensation between wet and dry states indicated that under wet conditions fabrics felt more texturized compared to dry, causing reductions in pleasantness sensation. 
Wetness perception and surface texture

Due to the critical impact of tactile sensitivity on wetness perception we hypothesised that fabric texture properties and/or sensation could affect wetness perception through changes in skin tactile responses, such as stickiness sensation. In particular, we expected the wet smoother surfaces to cause higher stickiness sensation; the latter likely due to the higher number of contact points between the skin and the fabric, also causing higher skin displacement compared to the rougher surfaces.

Two of the eight experimental fabrics (LPM6 and MP2) presented different wetness perception responses compared to those fabrics presenting same water content (Fig 3). However, these differences could not be attributed to the measured fabric texture propertiy, per se. For instance, in the low thickness group (L), LPM6 was perceived significantly dryer than LCO4, LP3 and LP6, and although LPM6 was rougher than LCO4 and LP3 it was not rougher than LP6 (Table 1). Additionally, LCO4, LP3 and LP6 presented the same wetness perception scores (Fig 3), despite differences in surface texture. Similarly, in the high thickness group $(\mathrm{H})$, despite the surface texture of samples HP4 and HP15 was considerably different, 3.65 versus 15.3 respectively, no significant differences in wetness perception were observed (Fig 3). On the contrary in M (medium thickness group) wetness perception was significantly different between sample MP2 and MP3 (Fig 3) even though the difference in surface texture was quite small (1.9 versus 2.7, respectively). 
The above-mentioned observations are validated by the lack of correlations between stickiness sensation and surface texture as well as between wetness sensation and surface texture, indicating that changes in stickiness sensation and related wetness cannot be attributed to the surface texture parameter (i.e. surface roughness), measured with the Kawabata Evaluation System. It could be argued that the ST of the samples was not different enough to show its influence on stickiness sensation (between 2.6 and 6.4, except for HP15 in which it was 15.3). However, despite these small differences in ST the participants could sense significant differences in texture across the fabrics, suggesting that the Kawabata Evaluation System is not sensitive enough or not as sensitive as humans.

The significant differences observed within the same thickness group, i.e. between fabrics presenting same absolute $\left(\mu \mathrm{L} \cdot \mathrm{mm}^{-2}\right)$ and relative water content $\left(\mu \mathrm{L} \cdot \mathrm{mm}^{-3}\right)$, suggest that certain surface and texture properties might still affect the mechanical interaction between the skin and the fabric under wet conditions. However, in order to asses this, other measures different from KES, or more suitable means able to characterise fabric surface properties are needed.

In L (low thickness group), LPM6 performed as the best fabric in terms of wetness perception, being perceived as drier than LCO4, LP3 and LP6. Fabric LPM6 is a polyester material in which the fibre cross-section consists of a series of closely spaced channels (either tetra- or hexa-channels) increasing the total surface area and facilitating 
the capillary action. As such, the theory is that moisture is wicked along the fibre surface and spread across a wider fabric surface area, enhancing evaporation. Hence, in LPM6 the faster evaporation rate should have resulted in a higher local skin temperature drop from baseline, however this was not the case (Fig 3, panel A). Therefore, it is possible that the fuzzy structure of LPM6 reduced the skin adhesiveness during the application process, causing lower stickiness sensation (Fig 5) and wetness perception. On the other hand, differences in fibre type between LCO4 (cotton) and LP6 or LP3 (polyester), did not determine changes in surface texture such as to affect stickiness sensation and related wetness perception. Similarly, the substantial difference in surface texture between LP3 and LP6, as well as between HP4 and HP15 did not influence stickiness sensation or wetness perception. These two pairs of fabrics had different knit structure, but the yarn type was identical. The latter suggests that changing the knit structure might not affect the mechanical interaction between the skin and the fabric; however the effect of yarn shape was not investigated. Finally, MP2 was perceived significantly wetter and stickier than MP3. Even in this case these differences could not be attributed to the texture parameters measured by the Kawabata Evaluation System, given that the difference in surface texture was minimal (1.9 in MP2 versus 2.7 in MP3). Nevertheless, it is likely that the silicon treatment applied to MP2 caused higher adhesiveness with the skin under wet conditions, resulting in higher stickiness sensation and wetness perception. 
The role of tactile-sensitivity on fabric wetness perception

Unlike fabric surface texture, stickiness sensation was related to wetness perception. When the fabric and/or the skin is wet the higher adhesiveness (Nacht et al. 1981) increases the frictional force between the two surfaces (Nacht et al. 1981; Kenins 1994). In normal wear conditions the higher adhesiveness occurs in response to the increase in the size of the cells of the stratum corneum, when it is wet, which result in a higher number of contact points between the fabric and the skin (Gwosdow et al. 1986). This higher frictional force may cause greater skin displacement, sensed by the cutaneous mechanoreceptors as higher stickiness and perceived as greater wetness. Skin stickiness sensation was not related to fabric water content, likely because the experimental fabrics were tested under the same saturation level (50\%). Additionally, given that the same pressure condition was applied, the individual weight of the fabric, pressing on the skin, did influence stickiness sensation. Based on this, the skin mechanical stimulation when in contact with a wet material might be affected by various factors and it seems not as straight forward to identify a single parameters triggering stickiness sensation.

The contribution of tactile-sensitivity to wetness perception is corroborated by the significantly different responses between high and low pressure conditions, also observed under static conditions (Raccuglia et al. 2016). In fact, almost all of the experimental fabrics were perceived significantly wetter under higher compared to lower pressure conditions. Conversely, Filingeri et al. (2014b) observed a diminished 
wetness perception when increasing the contact pressure of the wet stimulus applied to the skin. In Filingeri's et al. study (2013) a significantly higher contact pressure was applied compared to the current study, (10000 Pa versus $260 \mathrm{~Pa}$ ) suggesting that there might be a U-shape relationship between wetness perception and contact pressure. However, a contact pressure of 260 Pa seems more realistic for the current applications, therefore reducing the fabric-to-skin pressure is recommended for the design of clothing with reduced wetness perception.

The significant relation between wetness perception and stickiness sensation as well as the role of fabric-to-skin pressure, indicate that wetness perception can be manipulated by changing the tactile stimulation of the skin. In practice, using fabrics with reduced stickiness sensation features, together with the use of lightweight materials can help the clothing industry in designing garments with reduced moisture discomfort.

The role of thermo-sensitivity on fabric wetness perception

In line with the earlier results obtained in static applications (Raccuglia et al. 2016), a significant relation was observed between wetness perception and local skin temperature drop. With the increase in fabric water content, the drop in local skin temperature also increases, the latter sensed as higher cooling and associated with greater wetness perception. Fabric water content is mainly influenced by fabric thickness (Raccuglia et al. 2016). Because of the important relation between these two 
parameters, fabric thickness has been indicated as a critical factor affecting wetness perception under static fabric-to-skin contact (Raccuglia et al. 2016).

In the current dynamic condition no significant relations were observed between wetness perception and fabric thickness. However, when examining the model, it is evident that the lack of relation was mainly caused by two fabrics: LPM6 and MP2. These two fabrics did not fit in the regression line, because of their significantly higher and lower stickiness sensation, respectively. The latter suggests that under dynamic skin contact fabric thickness can predict fabric wetness perception only when considered in combination with stickiness sensation. This was shown by the multiple regression analysis which indicated stickiness sensation and fabric thickness as valid predictor of wetness perception $\left(r^{2}=0.86\right)$.

Because of the correlation between local skin temperature drop an thickness/water content (Fig 4) a similar prediction model is obtained when replacing the variable ‘thickness' with 'local skin temperature drop'. Indeed, when using local skin temperature drop, instead of thickness, together with stickiness sensation as variables, a stronger prediction model is obtained $\left(r^{2}=0.89\right)$. This means that local skin temperature drop is as better predictor than fabric thickness in dynamic conditions, pointing towards the temperature drop being the mechanism of action, and fabric thickness showing an effect due to its correlation with this, based on water content for evaporative cooling. 
Pleasantness and texture sensation of dry and wet fabrics

Pleasantness and comfort are criteria commonly used by the users when selecting fabrics and clothing. Pleasantness was significantly reduced when fabric texture sensation increased (Fig 10). The significant relation between texture sensation and pleasantness indicates that fabric texture is an important parameter to consider in terms of clothing acceptability, in addition to wetness perception and thermal comfort. Interestingly, under wet state fabric texture sensation significantly increased compared to dry and resulted in a concomitant reduction in fabric pleasantness sensation. In line with this, Gwosdow et al. (1986b) indicated that fabrics feel more textured as skin wetness rose above 20\%. Therefore, judgements of fabric texture and associated pleasantness can change in relation to the hydration state of the skin and/or fabric moisture content. As such, evaluations of fabric/clothing texture and related acceptability should be conducted under both dry and wet conditions.

Due to practical reasons and to prevent the effect of personal, environmental and clothing factors on the outcomes, in this study we studied comfort-related properties of fabrics only at the ventral forearm. We speculate that the current results could show a similar trend at different body regions; however it is unknown to what extent the outcomes will be different across the body. For instance, according to the mechanisms underlying skin wetness perception, body regional differences would likely depend on human sensorial factors, such as thermal and tactile sensitivity, as well as anatomical 
factors, i.e. hair distribution and differences between glabrous/non-glabrous skin. In terms of an overall garment, clothing factors, such as the air gap between the skin and the fabric as well as clothing fit (both influencing the level of fabric-to-skin contact) represent additional variables that would influence wetness perception responses across the body. Hence, future researches are necessary to understand how this initial results relate to an overall garment.

\section{Conclusion}

We studied textile and non-textile factors contributing to wetness perception of fabric treated with the same relative water content $\left(\mu 1 \cdot \mathrm{mm}^{-3}\right)$ and in dynamic skin contact conditions.

Local skin temperature drop/fabric thickness and stickiness sensation can predict wetness perception of fabrics in dynamic contact with the skin, whereas fabric surface texture measured by the Kawabata Evaluation System had no impact at all. The latter indicates that the Kawabata Evaluation system fails to predict stickiness sensation of wet fabrics, commonly assumed to be associated with fabric texture. Thus a different way to define fabric texture may be needed in order to represent this link (stickiness and texture).

Sensations of pleasantness are highly influenced by ST (surface texture measured by KES) and even more by the sensation of fabric texture (i.e. roughness and smoothness): 
as ST and the roughness of the fabric sensed on the skin increase, pleasantness sensation diminishes. Additionally, in wet conditions fabrics are sensed more texturized, this resulting in a concomitant reduction in pleasantness sensation. Therefore, assessment of fabric pleasantness and acceptability in relation to fabric texture properties are recommended under both dry and wet conditions.

By identifying the textile and clothing parameters influencing skin wetness perception and related discomfort, this study provides fundamental knowledge for the design of clothing with reduced moisture discomfort features. Nevertheless, future researches are necessary to understand how this initial result related to an overall garment.

Conflict of interests: none. 


\section{References}

Alimaa D, Matsuo T, Nakajima M, Takahashi M (2000) Sensory Measurements of the Main Mechanical Parameters of Knitted Fabrics. Text Res J 70:985-990.

Bentley IM (1900).The Synthetic Experiment The American Journal of Psychology Vol. 11, No. 3, pp. 405-425.

Bergmann Tiest WM, Kosters ND, Kappers AML, Daanen HAM (2012) Haptic perception of wetness. Acta Psychol (Amst) 141:159-63.

Cardello A V., Winterhalter C, Schutz HG (2003) Predicting the Handle and Comfort of Military Clothing Fabrics from Sensory and Instrumental Data: Development and Application of New Psychophysical Methods. Text Res J 73:221-237.

Filingeri D, Fournet D, Hodder S, Havenith G (2014a) Why wet feels wet? A neurophysiological model of human cutaneous wetness sensitivity. J Neurophysiol 112:1457-69.

Filingeri D, Havenith G (2015) Human skin wetness perception: psychophysical and neurophysiological bases. Temperature 2:86-104.

Filingeri D, Redortier B, Hodder S, Havenith G (2014b) Thermal and tactile interactions in the perception of local skin wetness at rest and during exercise in thermo-neutral and warm environments. Neuroscience 258:121-30. doi: 
10.1016/j.neuroscience.2013.11.019

Fukazawa T, Havenith G (2009) Differences in comfort perception in relation to local and whole body skin wettedness. Eur J Appl Physiol 106:15-24.

Gwosdow AR, Stevens JC, Berglund LG, Stolwijk JAJ (1986) Skin Friction and Fabric Sensations in Neutral and Warm Environments. Text Res J 56:574-580.

Houdas Y, Ring E (1982) Human body temperature - Its measurement and regulation. Plenum Press, New York, NY.

Jeon E, Yoo S, Kim E (2011) Psychophysical determination of moisture perception in high-performance shirt fabrics in relationtosweating level. Ergonomics 54:576-586.

Kawabata S (1980) The standardization and analysis of hand evaluation. Textile Machinery Society of Japan, Osaka

Kenins P (1994) Influence of Fiber Type and Moisture on Measured Fabric-to-Skin Friction. Text Res J 64:722-728.

Li Y (2005) Perceptions of temperature, moisture and comfort in clothing during environmental transients. Ergonomics 48:234-48.

Nacht S, Close J-A, Yeung D, Gans EH (1981) Skin friction coefficient: changes induced by skin hydration and emollient application and correlation with perceived 
skin feel. j Soc Cosmet Chem 32:55-65.

Niedermann R, Rossi RM (2012) Objective and subjective evaluation of the human thermal sensation of wet fabrics. Text Res J 82:374-384.

Parsons K (2002) Human Thermal Environments: The Effects of Hot, Moderate, and Cold Environments on Human Health, Comfort and Performance, Second Edition. CRC Press

Raccuglia M, Hodder S, Havenith G (2016) Human wetness perception in relation to textile water absorption parameters under static skin contact.

Schlader ZJ, Stannard SR, Mündel T (2010) Human thermoregulatory behavior during rest and exercise - a prospective review. Physiol Behav 99:269-75.

Sivamani RK, Goodman J, Gitis N V., Maibach HI (2003) Coefficient of friction: tribological studies in man - an overview. Ski Res Technol 9:227-234.

Sular V, Okur A (2007) Sensory evaluation method for tactile properties of fabrics. J Sens Stud 22:1-16.

Sweeney MM, Branson DH (1990a) Sensorial Comfort: Part I: A Psychophysical Method for Assessing Moisture Sensation in Clothing. Text Res J 60:371-377.

Sweeney MM, Branson DH (1990b) Sensorial Comfort: Part II: A Magnitude 
Estimation Approach for Assessing Moisture Sensation 1. Text Res J 60:447-452.

Tang KPM, Kan CW, Fan JT (2014) Assessing and predicting the subjective wetness sensation of textiles: subjective and objective evaluation. Text Res J 85:838-849. 